\title{
COMPARISON OF VANADIUM OXIDE CATALYSTS FOR SYNTHESIS OF BENZENE: BENZENE PURITY, YIELDS AND RECONDITIONING METHODS
}

\author{
T. B. ENERSON, ${ }^{1}$ HERBERT HAAS, ${ }^{1}$ KAVEH ZARRABI ${ }^{2}$ and R. L. TITUS ${ }^{3}$
}

\begin{abstract}
This study compares vanadium oxide catalysts from three different sources: Noakes $(N)$, Harshaw Chemical (H) and Kh. Arslanov at the St. Petersburg State University, Russia (R). The catalysts are used to convert acetylene to benzene in the last step of benzene synthesis. The organic purity of benzene in all three catalysts is high; $99.91-99.93 \%$ for $(\mathrm{N})$ and $(\mathrm{H})$ and $99.87 \%$ for $(\mathrm{R})$. The benzene yields range from 90.0 to $94.3 \%$. $(\mathrm{N})$ averaged $92.6 \%$, (H) averaged $91.1 \%$ and $(\mathrm{R})$ averaged $92.0 \%$. A conversion residue in the catalysts was analyzed for ${ }^{13} \mathrm{C}$ and found to be isotopically lighter relative to acetylene by $-2.2 \%$ for $(\mathrm{N})$ and $(\mathrm{H})$ and $-3.9 \%$ for $(\mathrm{R})$. Benzene yields were studied on different reconditioning methods applied to all catalysts: heating to $400^{\circ} \mathrm{C}$ in air averaged $92.3 \%$; the same temperature with a half and half mixture of $\mathrm{O}_{2}$ and Ar averaged $91.9 \%$, adding a half and half mixture of $\mathrm{H}_{2}$ and $\mathrm{Ar}$ at $200^{\circ} \mathrm{C}$ to the end of this treatment averaged $91.8 \%$. Based on this research, the obvious difference seen between the catalysts is in their trace by-products.
\end{abstract}

\section{INTRODUCTION}

The majority of conventional radiocarbon dating laboratories use the liquid scintillation counting (LSC) method for detecting the abundance of the ${ }^{14} \mathrm{C}$ isotope. The carbon content of samples is converted to benzene, which is the scintillation counting medium. The final step in the benzene synthesis process is the joining of three acetylene molecules to one benzene molecule (Noakes, Kim and Akers 1967). Two types of catalysts are being used for this process: a chromium-coated catalyst (used mostly in Europe) and a vanadium oxide catalyst (used mostly in the United States and Russia). In the 1980s, production of the vanadium oxide catalyst ceased for economic reasons, and in Europe the chromium catalyst is no longer available in its most suitable formula due to environmental considerations. Consequently, an acute shortage of these catalysts now exists. In the recent past, the only source for freshly produced vanadium oxide catalyst has been a laboratory in St. Petersburg, Russia.

Conversion of carbon to benzene must occur without isotope fractionation. A conversion technique with $100 \%$ efficiency would fulfill this condition. In reality the catalytic conversion of acetylene to benzene proceeds with a yield between 87 and $94.3 \%$ in our laboratory. Isotope fractionation is a problem with the lower-range yields.

LSC is based on the conversion of decay energy of radioactive nuclides into photon energy by the scintillator (Kessler 1991). The efficiency of this process influences the accuracy of the rate-ofdecay measurement. This efficiency is controlled by the chemical purity of the benzene. The catalytic manufacture of benzene is a potential source of impurities in the benzene (Noakes, Kim and Stipp 1965). These impurities may have a quenching effect on scintillation counting.

\section{Handling of Catalysts}

Vanadium oxide catalysts can be reused numerous times (up to 50 in our lab). After each use the catalyst must be "reconditioned", i.e., residue from the previous application must be removed and the vanadium oxide must be returned to the ideal oxidation state of four and five (Coleman 1973). At the DRI laboratory, we do not follow the common method of removing the catalyst from its vessel for reconditioning in air. Instead, the catalyst is heated to $400^{\circ} \mathrm{C}$ in its own vessel and is reacted with a 1:1 ratio of $\mathrm{O}_{2}$ and $\mathrm{Ar}$ to combust the residual organic compounds left from the catalytic conversion.

\footnotetext{
${ }^{1}$ Desert Research Institute, Water Resource Center, P. O. Box 19040, Las Vegas, Nevada 89119 USA

${ }^{2}$ Community College of Southern Nevada, Dept. of Chemistry, 6375 West Charleston Blvd., Las Vegas, Nevada 89102 USA

${ }^{3}$ University of Nevada, Las Vegas, Dept. of Chemistry, 4505 South Maryland Parkway, Las Vegas, Nevada 89154 USA
} 
Because the vessel has a finite volume, it must be evacuated and fresh $\mathrm{O}_{2} / \mathrm{Ar}$ mixture added. This is done ten times, or at least 5 gas exchanges after there is no more steam seen during the evacuation of the vessel. The catalyst is cooled to $200^{\circ} \mathrm{C}$ and is given two exposures with a $1: 1$ ratio of $\mathrm{H}_{2}$ and Ar. With this procedure the catalyst pellets are left in the vessel and only high purity gases are used. The use of medical grade oxygen, ultra high purity argon and high grade hydrogen keep the catalyst from reacting with potentially contaminated laboratory air. The step of removing the catalysts pellets and putting them back in the vessel after conditioning is eliminated, and the risk of introducing contaminants into the vessel or on the pellets is reduced.

The catalyst is used on samples of acetylene ranging from 0.4 to $6.0 \mathrm{~L}$. Accordingly, we use vessels filled with $30-100 \mathrm{~g}$ catalyst. The acetylene is reacted with the catalyst at 330 mbar pressure. All samples are left in the vessel overnight.

Greaseless stopcocks in the acetylene conversion system are used to avoid traces of silicone or organic stopcock grease in the benzene. Before converting to greaseless stopcocks we detected these contaminants (Haas 1995). The catalyst vessel also has no grease joints and has a large bore valve to allow easy filling of pellets.

For extraction of the benzene, the vessel is heated to $100^{\circ} \mathrm{C}$ for a period of $3 \mathrm{~h}$. One hour into the extraction, a valve downstream from the freeze trap is opened to monitor and remove any residual pressures in the extraction system. A final vacuum transfer into a removable storage vial assures a contamination-free removal of the benzene from the system. By following these procedures we maintain a high purity benzene.

For this research three different methods of reconditioning were applied. This was done to offer comparative results for laboratories following different procedures. The following symbols are used on all graphs presented in this research:

- No prime (N, H, R): Recondition with $\mathrm{O}_{2}$ and Argon at $400^{\circ} \mathrm{C}$ followed by $\mathrm{H}_{2}$ and Argon at $200^{\circ} \mathrm{C}$.

- One prime ( $\left.\mathrm{N}^{\prime}, \mathrm{H}^{\prime}, \mathrm{R}^{\prime}\right)$ : Recondition with $\mathrm{O}_{2}$ and Argon at $400^{\circ} \mathrm{C}$.

- Two prime (N", H", R"): Recondition with air at $400^{\circ} \mathrm{C}$.

\section{Major Impurities in Synthesized Benzene}

The source of carbon for this research was $\mathrm{CO}_{2}$ from a pressurized tank. $\mathrm{CO}_{2}$ was passed through the gas cleaning traps installed in the combustion line, after which it showed very little residual pressure when frozen in liquid nitrogen ( $<5$ millitorrs $[\sim .67 \mathrm{~Pa}]$ ). Twelve liters $\mathrm{CO}_{2}$ was converted to acetylene, which was divided into three equal parts of $2 \mathrm{~L}$ each, one part for each of the three catalyst types used in this research. The gas chromatography (GC) analysis on the benzene samples were performed using a Hewlett Packard 5770 with a flame ionization detector (FID). The gas chromatography (Fig. 1a,b) of synthesized benzene samples revealed presence of organic impurities. These organic impurities were quantified by using GC/MS (gas chromatograph/mass spectrometer). Figure $1 \mathrm{c}$ is an overview of all analyses made on benzene extracted from catalysts with different reconditioning methods. It shows the concentration of the corresponding major impurities, which are toluene and ethylbenzene. The GC/MS analysis were performed using a Hewlett Packard 5880. All GC analysis used a $30 \mathrm{M} \times 0.32 \mathrm{~mm}$ DB-1 column. 

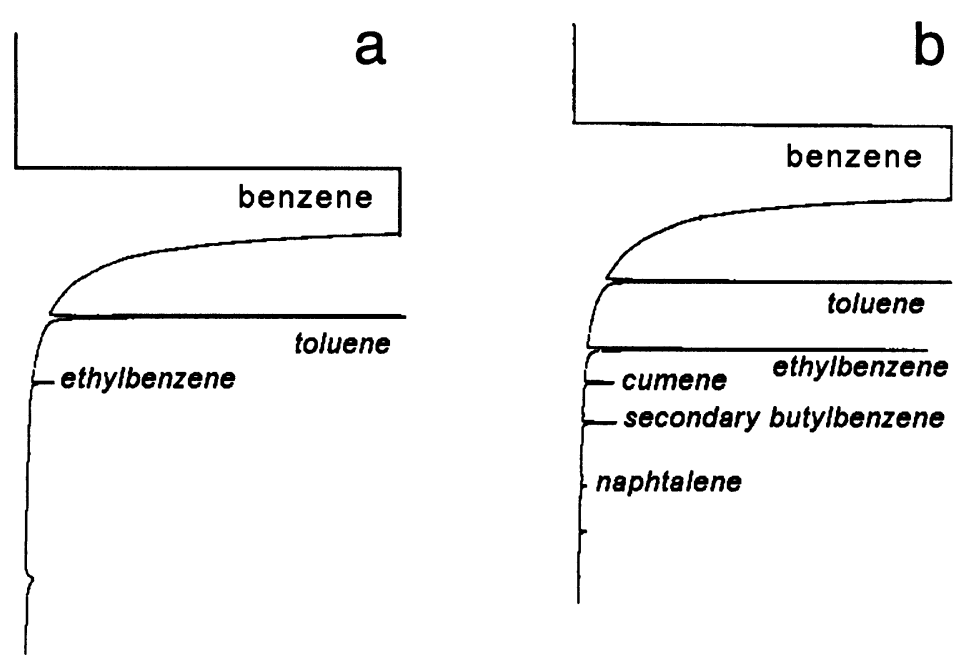

Fig. 1. Gas chromatograms of benzene from (a) Harshaw and (b) Russian catalysts. Harshaw and Noakes showed similar spectra.

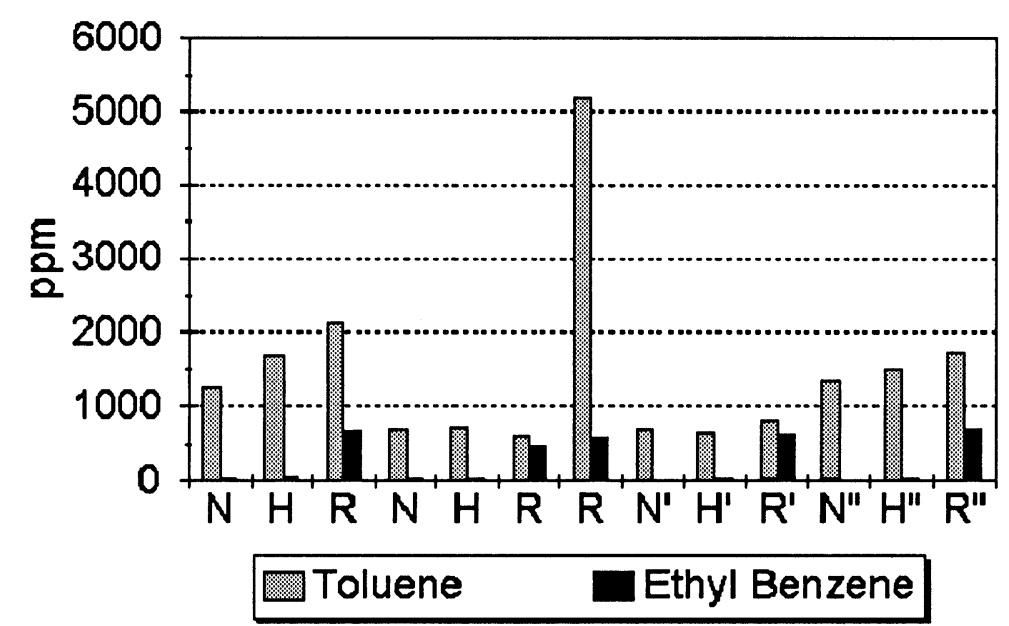

Fig. 1c. Bar graph showing the major impurities of toluene and ethylbenzene found in each of the catalysts by using GC/MS. This graph also shows the effect of the different reconditioning methods used on the catalysts.

\section{Water Content in Benzene}

Figure 2 shows the water content found in the benzene samples. Water has been reported to have quenching effects (Kessler 1991). Our research found this quenching effect to be minimal. The quenching was studied by taking one large source of carefully dried benzene using a molecular sieve. This benzene was split into two portions, one that was untouched and one that was saturated with water. Those two end members and mixtures of various proportions were prepared and counted. The difference for all samples counted were insignificant at the level of a 2- $\sigma$ counting error. 
The water content of the synthesized benzene was determined using a modified hydride reaction method (Seidov 1981). A known volume of the benzene was injected into a slurry of sodium hydride in toluene and the volume of the hydrogen generated was measured.

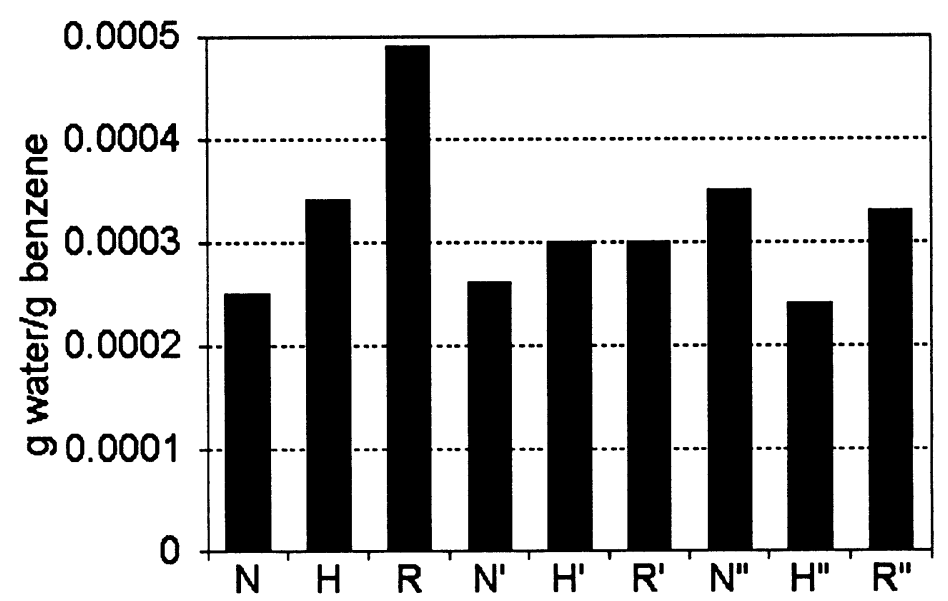

Fig. 2. Bar graph showing the amount of water found in the benzene from the catalysts reconditioned in the three different methods

\section{Trace Impurities in Benzene as a Function of Reconditioning Method}

Figures $3 a, 3 b$ and $3 c$ show how the concentration of impurities vary with the no prime, one prime and two prime reconditioning techniques used on the catalysts. In addition to the major impurities the (R) catalyst also produces trace amounts of other hydrocarbons. These hydrocarbons are cumene, secondary butylbenzene and naphthalene. With our equipment, trace impurities were not detected in the benzene extracted from $(\mathrm{H})$ and $(\mathrm{N})$.

\section{Extractable Residue in Catalysts}

After benzene extraction, the catalysts were studied in order to determine the nature of the unrecovered sample fraction. The conversion from acetylene to benzene yields mostly benzene (87-94.3\%) and a portion of $5.7-13 \%$ remains as a residue in the catalyst. This residue is found to have isotope fractionation (see Table 1 and following discussion). We believe that high molecular weight compounds and elemental carbon are produced by the catalyst (Coleman 1973). To study this missing sample fraction each of the catalysts was split into two equal parts. One part went for chemical analysis, the other for combustion of the organic residual.

The determination of the amounts of methylene chloride soluble compounds remaining on the catalyst after the removal of the benzene (Fig. 4) was performed by placing the catalyst sample in a Soxhlet apparatus and extracting the residue with methylene chloride for $c a .3 \mathrm{~h}$. The extract was then concentrated to $1 \mathrm{~mL}$ for GC/MS analysis using a Kurdana-Danish (KD) apparatus. Dodecane was added as an internal standard for GC/MS analysis. The concentration of the dodecane internal standard in the sample was $375 \mathrm{ng} \mu \mathrm{L}^{-1}$.

The second half of the catalyst was heated in its own vessel to $400^{\circ} \mathrm{C}$ in the presence of $\mathrm{O}_{2}$ to oxidize residual carbon to $\mathrm{CO}_{2}$. This procedure is the usual initial step of reconditioning. Water and $\mathrm{CO}_{2}$ were collected by pumping the gas mixture through dry ice and liquid nitrogen traps. The water was measured crudely for an estimate on a mass balance, i.e., the amount of carbon that came from 

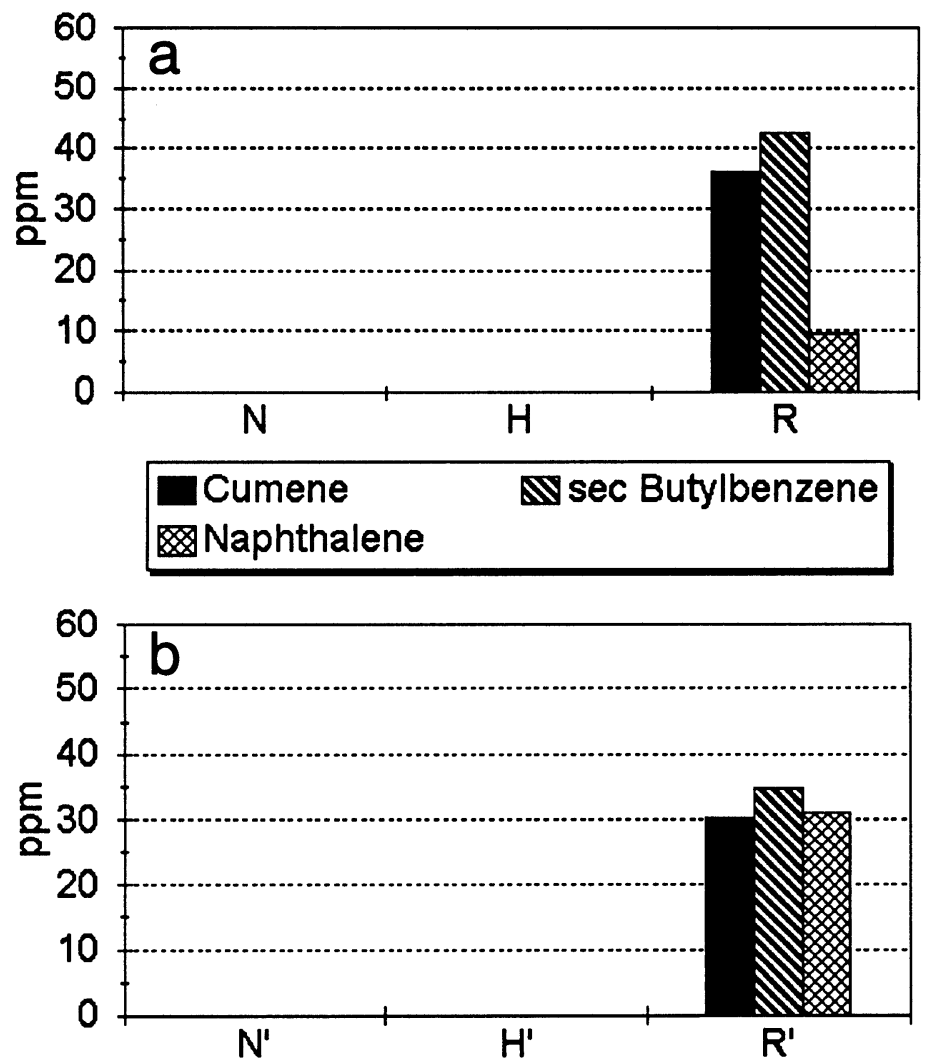

Cumene $\mathbf{N e c}$ Butylbenzene Naphthalene
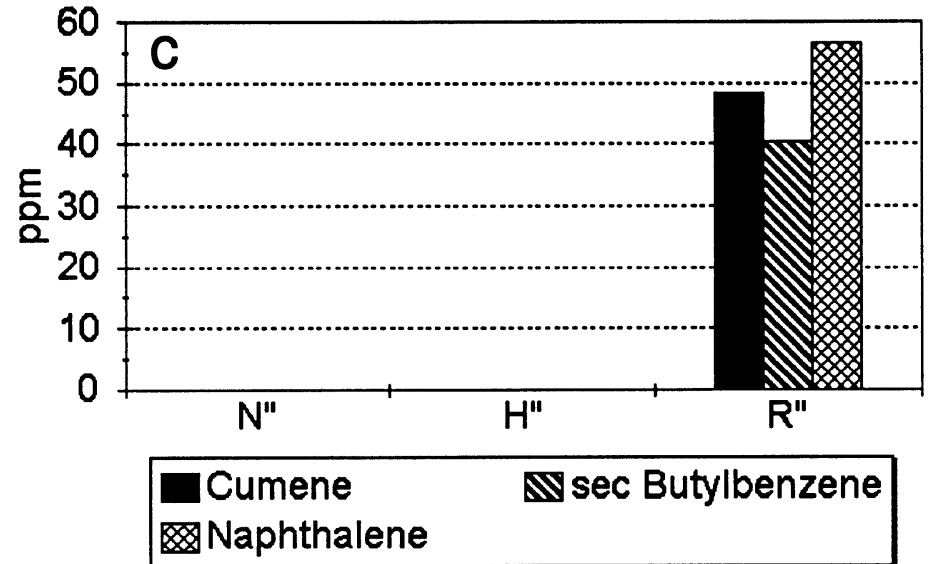

Fig. 3. Trace impurities in the catalysts treated with (a) $\mathrm{O}_{2}$ and argon at $400^{\circ} \mathrm{C}$ followed by $\mathrm{H}_{2}$ and argon at $200^{\circ} \mathrm{C}$; (b) $\mathrm{O}_{2}$ and argon at $400^{\circ} \mathrm{C}$; (c) air at $400^{\circ} \mathrm{C}$. There are no detectable trace impurities in either the Harshaw or Noakes catalysts. 

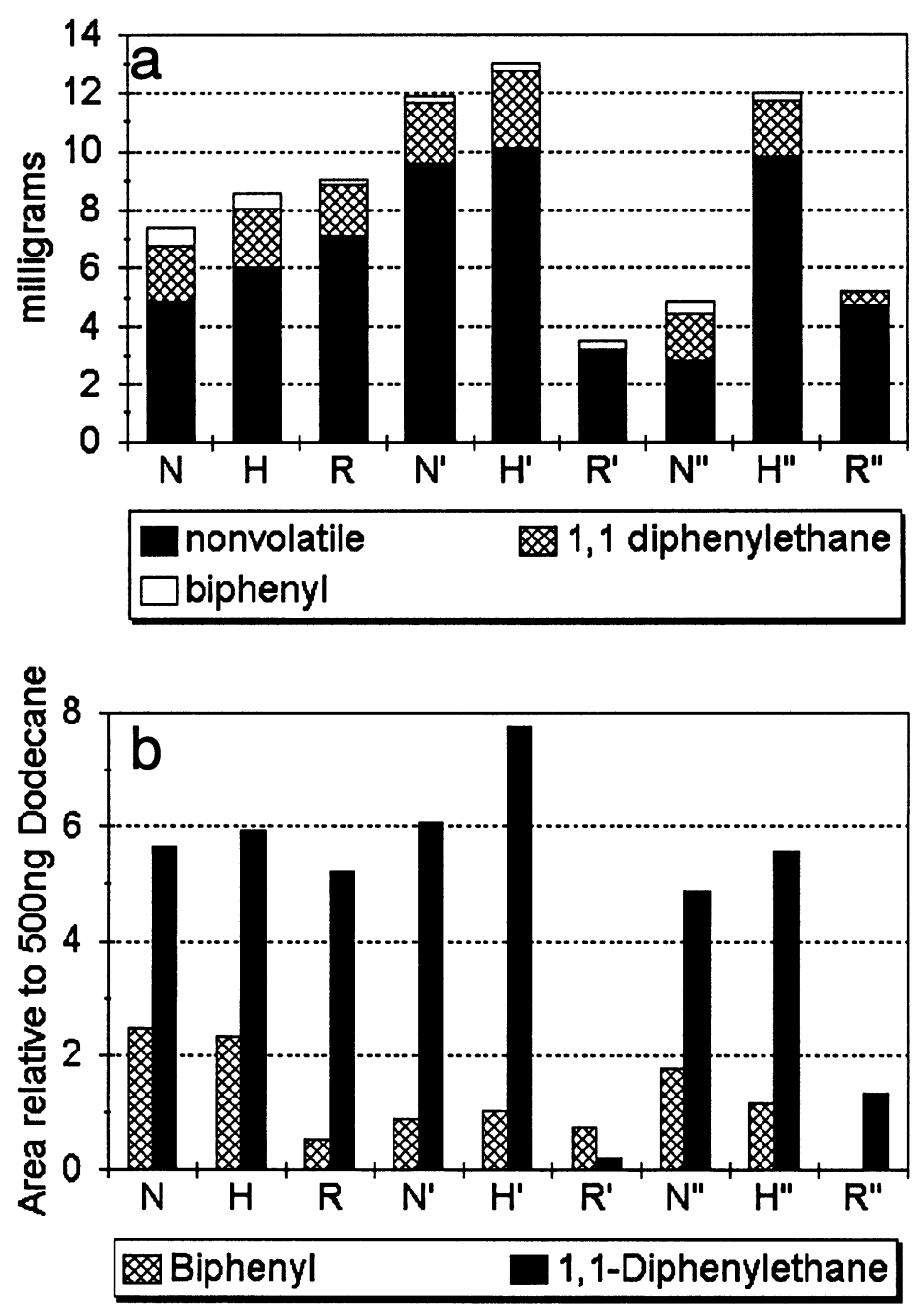

Fig. 4 (a) Bar graph showing the amounts of residual compounds extracted from the catalysts after benzene extraction. The effects of the different reconditioning methods can also be seen. (b) Graph showing the relationship between the 1,1-Diphenyl ethane and biphenyl for each of the catalysts.

hydrocarbon or elemental carbon. This procedure will be improved for better quantitative analysis of the water in future experiments. $\mathrm{CO}_{2}$ was transferred to a well-calibrated manifold for gas volume measurements. These volumes showed a recovery of $52-63 \%$ of the missing sample fraction. Because the catalyst was combusted in its own vessel, there was a finite volume of $\mathrm{O}_{2}$. The percentages of recovery do not take into account the production of $\mathrm{CO}$ that could not be collected. Future research will correct this by passing a constant flow of $\mathrm{O}_{2}$ over the heated catalyst.

$\mathrm{CO}_{2}$ was analyzed for isotope fractionation between the carbon source and the combusted residual. All $\delta^{13} \mathrm{C}$ ratios measured on the residual fraction are isotopically lighter than the carbon source for these experiments. The measured values are shown in Table 1.

A fractionation effect on the benzene must be assumed. Fractionation would result in a heavier isotope ratio and the difference from the carbon source would be considerably smaller because of the 
larger benzene mass. Using average benzene yield factors and the standard age calculation method leads to an estimated error in ${ }^{14} \mathrm{C}$ dates, shifted by $0.22-0.4 \%$, which corresponds to an age shifted by $4-7 \mathrm{yr}$ to younger ages.

TABLE 1. Fractionation from the Catalysts (Differences in \%o)

\begin{tabular}{cccccc}
\hline $\mathrm{R}^{\prime \prime}$ & $\mathrm{H}$ & $\mathrm{H}^{\prime}$ & $\mathrm{H}^{\prime \prime}$ & $\mathrm{N}$ & $\mathrm{N}$ \\
\hline-3.94 & -2.76 & -1.60 & -2.20 & -2.25 & -2.13 \\
\hline
\end{tabular}

\section{Routine Application of Three Catalysts at the DRI Laboratory}

To compare this planned study to routine laboratory data, we show Figure 5, benzene yields from the $\mathrm{N}, \mathrm{H}$ and $\mathrm{R}$ catalysts. Table 2 shows the values for the high and low yields and the overall average yield for each of the catalyst.

TABLE 2. Averages and High/Low Yields for Each of the Catalysts Over a Four-Month Period of Routine Lab Use at DRI Using the No-Prime Reconditioning Method

\begin{tabular}{lccc}
\hline & $\mathrm{N}$ & $\mathrm{H}$ & $\mathrm{R}$ \\
\hline Lowest & $87.3 \%$ & $86.7 \%$ & $87.0 \%$ \\
Highest & $92.7 \%$ & $93.6 \%$ & $96.5 \%$ \\
Average & $90.3 \%$ & $91.3 \%$ & $91.4 \%$ \\
\hline
\end{tabular}

The figures also show the amount of uses from the same catalyst with no prime $\left(\mathrm{O}_{2}\right.$ and $\left.\mathrm{H}_{2}\right)$ reconditioning, which is the standard technique used at DRI. The slope of the trend line is positive for $R$ and slightly negative for $\mathrm{N}$ and $\mathrm{H}$. Clearly, yields are predictable and steady over 30 or more uses for each of the catalysts. Also, the catalysts did not show any significant yield variation with different carbon sources. This is important because some sample types such as bone collagen or humates do not produce pure $\mathrm{CO}_{2}$ and presumably acetylene gas. Prior to this research it was suspected to have a direct relationship with benzene yields.

\section{CONCLUSION}

There is no significant difference between the three vanadium oxide catalysts. Trace impurities produced by the Russian catalyst are distinctly higher but it is not clear whether these impurities have an influence on scintillation counting (quenching). Water content of benzene is $<0.5 \%$ and was slightly higher in the Russian catalyst.

Different reconditioning methods have little impact on benzene yield and impurities. We therefore prefer a method that minimizes handling by allowing the reconditioning to take place inside the catalyst vessel, using pure tank gases and avoiding potentially contaminated laboratory air.

Failure to produce benzene with high, near $100 \%$ yields requires considering isotope fractionation between the benzene produced and an unrecovered residual fraction. Partial results of an analysis of the residual in the catalyst suggest a small but not negligible error in age dates, affecting mostly laboratories specializing in precision dating.

Ongoing research investigates the non-benzene byproducts in the catalyst. Trace impurities, including water, in the benzene will be studied for their impact on scintillation counting. Organic residue in the catalyst and fractionation resulting from low benzene yield will be part of this investigation. 


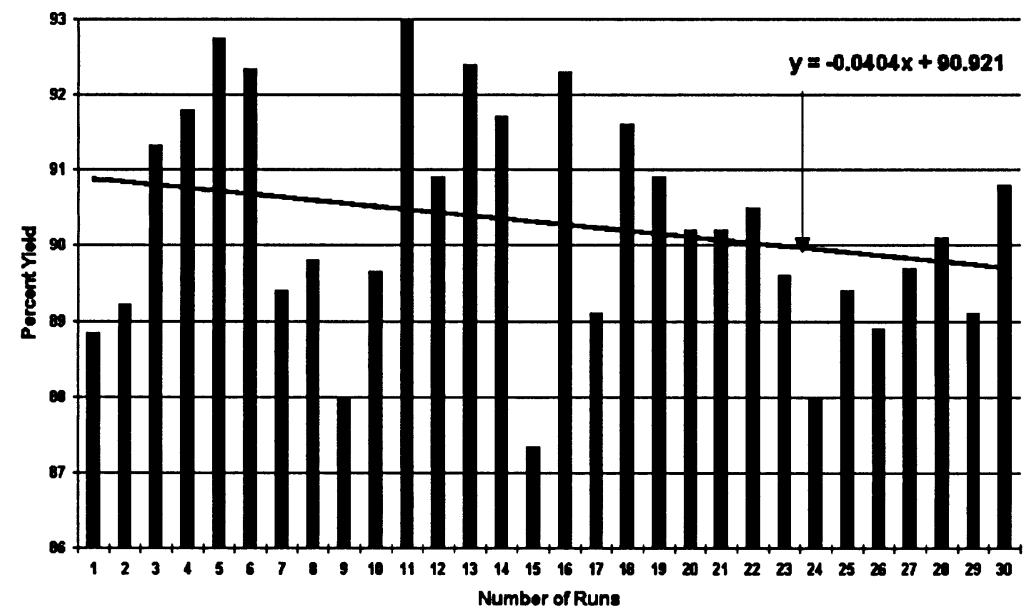

a
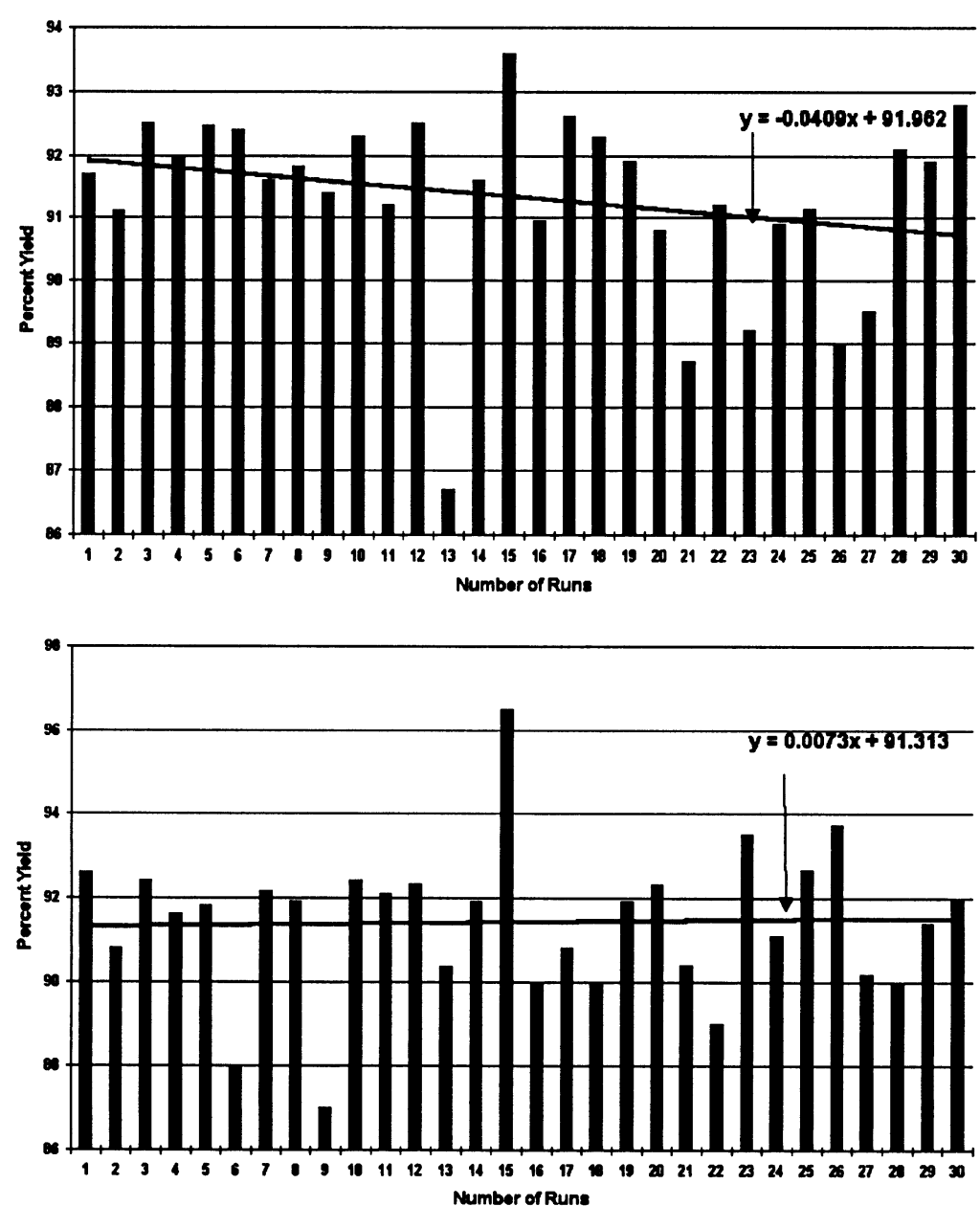

Fig. 5. Bar graphs showing the benzene yields of (a) the Noakes catalyst. (b) the Harshaw catalyst, and (c) the Russian catalyst 


\section{ACKNOWLEDGMENTS}

Research shown in this paper was carried out at Desert Research Institute, University of Nevada, Las Vegas, and Community College of Southern Nevada. The research was supported by the Nevada TREC (Teaching \& Research-Enhancement \& Collaboration) Program.

\section{REFERENCES}

Coleman, D. D., Liu, C. L., Dickerson, D. R. and Frost, R. R. 1973 Improvement in trimerization of acetylene to benzene for radiocarbon dating with a commercially available vanadium oxide catalyst (Abstract). In Rafter, T. A. and Grant-Taylor, T., eds., Proceedings of the 8th International Conference on ${ }^{14} \mathrm{C}$ Dating. Wellington, New Zealand, Royal Society of New Zealand: 158.

Haas, H. 1995 Change of calibration parameters in frequently used vials in benzene scintillation counting. In Cook, G. T., Harkness, D. D., Miller, B. F. and Scott, E. M., eds., Proceedings of the 15 th International ${ }^{14} \mathrm{C}$ Conference. Radiocarbon 37(2): 737-742.

Kessler, M. J. 1991 Applications of quench monitoring using transformed external standard spectrum (tSIE). In Ross, H., Noakes, J. E. and Spaulding, J. D., Liquid
Scintillation Counting and Organic Scintillators. Michigan, Lewis Publishing, Inc.: 343-364.

Noakes, J., Kim, S. and Akers, L. 1967 Recent improvements in benzene chemistry for radiocarbon dating. Geochimica et Cosmochimica Acta 31: 1094-1096.

Noakes, J. E., Kim, S. M. and Stipp, J. J. 1965 Chemical and counting advances in liquid scintillation age dating. In Radiocarbon and Tritium Dating: Proceedings of the 6th International Conference on Radiocarbon and Tritum Dating, Washington State University, Pullman, Wash., June 7-11, 1965. Oak Ridge, Tennessee, U.S. Atomic Energy Commission: 650-652.

Seidov, Sh. I., Shamilov, T. O., Gashinova, T. M. and Zarbalieva, R. A. Zh. 1981 Zhurnal Analiticheskoi Khimii 36(6): 1225-1228. 